\title{
Understanding Cultural Heritage Experts' Information Seeking Needs
}

\author{
Alia Amin, Jacco van Ossenbruggen, \\ Lynda Hardman \\ P.O. Box 94079 \\ 1090 GB Amsterdam \\ The Netherlands \\ firstname.lastname@cwi.nl
}

\author{
Annelies van Nispen \\ Digitaal Erfgoed Nederland (DEN) \\ P.O. Box 90407 \\ 2509 LK Den Haag \\ The Netherlands \\ annelies.vannispen@den.nl
}

\begin{abstract}
We report on our user study on the information seeking behavior of cultural heritage experts and the sources they use to carry out search tasks. Seventeen experts from nine cultural heritage institutes in the Netherlands were interviewed and asked to answer questionnaires about their daily search activities. The interviews helped us to better understand their search motivations, types, sources and tools. A key finding of our study is that the majority of search tasks involve relatively complex information gathering. This is in contrast to the relatively simple fact-finding oriented support provided by current tools. We describe a number of strategies that experts have developed to overcome the inadequacies of their tools. Finally, based on the analysis, we derive general trends of cultural heritage experts' information seeking needs and discuss our preliminary experiences with potential solutions.
\end{abstract}

\section{Categories and Subject Descriptors}

H.3.3 [Information Search and Retrieval]

\section{General Terms}

Human Factors

\section{Keywords}

cultural heritage experts, information seeking

\section{INTRODUCTION}

Cultural heritage is a vast domain consisting of museums, archives, libraries and (non)goverment institutions. Searching for information in this domain is often challenging because the sources are rich and heterogeneous, combining highly structured, semi-structured and unstructured information, combining authorized and unauthorized sources, and combining both text and other media (e.g. image

* Lynda Hardman is also affiliated with the Technical University of Eindhoven.

Permission to make digital or hard copies of all or part of this work for personal or classroom use is granted without fee provided that copies are not made or distributed for profit or commercial advantage and that copies bear this notice and the full citation on the first page. To copy otherwise, to republish, to post on servers or to redistribute to lists, requires prior specific permission and/or a fee.

JCDL'08, June 16-20, 2008, Pittsburgh, Pennsylvania, USA.

Copyright 2008 ACM 978-1-59593-998-2/08/06 ...\$5.00. and video). To perform their daily work, domain experts need to access and exploit cultural heritage information in its full richness. The specific information seeking needs of these experts remains, however, a scantly researched area. This paper reports on a user study that was motivated by the need to understand the information seeking behavior of cultural heritage experts. The results of this study are currently being used to improve the search tools developed in the context of the MultimediaN e-culture project [15].

Key findings of our study include, first, that experts' daily search tasks are dominated by a range of different (relatively complex and high level) information gathering tasks, while the tools tend to be geared towards support for (relatively simple and low level) fact finding tasks. Second, many search tasks require experts to use and combine results from multiple sources, while the tools typically provide access to a single source. Third, we found that direct communication as a means for information transfer is greatly valued by experts. Finally, we found that trust in the information source is an important aspect of experts' search activity.

The paper is structured as follows. After discussing related work, we describe the setup and analyze the results of the user study. The analysis includes an information task classification of the use cases reported by the participants, a classification of the type of information sources used in these use cases and an analysis of the underlying search tasks. We then discuss the extent to which current tools support the experts' search tasks and illustrate inadequate tool support with concrete examples given by experts during the interviews. Finally, we discuss design considerations based upon our experiences within the MultimediaN project.

\section{RELATED WORK}

The motivations behind searching information have been studied extensively $[1,3,5,12,13]$. Research by Broeder [1], extended by Rose et al. [13], found search motivations such as navigational search, informational search or resource finding. Their research is mainly based on analyzing logs when people use a search engine and a short survey. It is, thus, difficult to know the real search motivation. Choo et al. [5] took a different approach and monitored web browsing activities of people for two weeks and conducted an interview to check the participants' search motivations. They found that people have different modes of searching, where each mode has its own traits and search strategy [4].

Kellar et al. [8] compared previous research on information seeking task categories $[5,11,16]$ and proposed a taxonomy which gives a more thorough overview on the information tasks. They used this model to explain peoples' behavior on the web. In this study, we extended this taxonomy to discuss online as well as offline infor- 


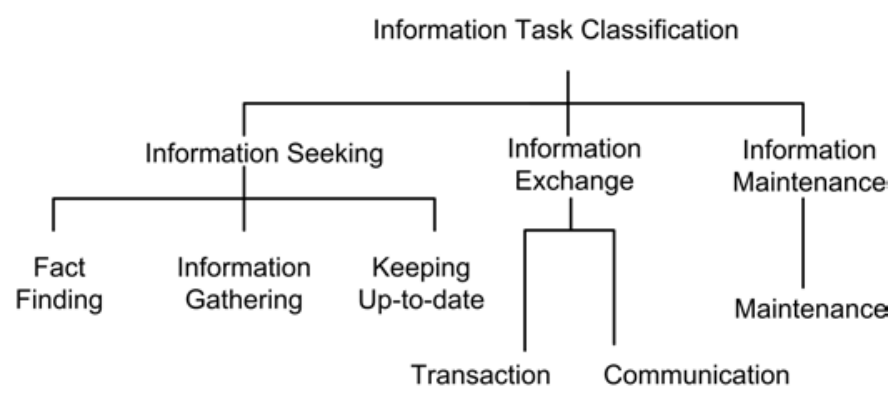

Figure 1: Classification of Information Tasks, adapted from [7].

mation task behavior for expert users. We use the following information tasks categories (adapted from [7, 8, 9], see Figure 1):

1. Fact Finding: users ask goal oriented and focused questions; they look for specific factual pieces of information.

2. Information Gathering: users carry out several search tasks to fulfill a higher level goal, such as writing a report, preparing an event, or collecting information to make a decision.

3. Keeping Up-to-date ${ }^{1}$ : this search task is generally not goaldriven, other than to "keep up-to-date", "just browsing", "see what is new or interesting", recreational searching or even "passing time".

4. Communication: an information exchange task, either faceto-face or through technology, such as email.

5. Transaction: an information exchange task, e.g., online auction, banking, downloading multimedia documents. Transactions are often associated with a user name and password combination.

6. Maintenance: a task which involves organizing information, e.g., updating bookmarks or organizing email in the appropriate folder.

To date, research on cultural heritage experts' search behavior is limited. A survey with 477 cultural heritage experts in the Netherlands on Collectiewijzer ${ }^{2}$ [19] usage reveals that experts think the Internet is becoming a more important information source. Experts say that they would use such system mainly to: do research, compare collections and look for potential items to borrow from other collections. The survey suggests that information seeking is an important part of experts' work. Unfortunately, it does not provide insights into experts' search behavior nor the kind of difficulties they experience when searching.

\section{USER STUDY SETUP}

The purpose of our study is to understand the search behavior, that is the information seeking tasks, of cultural heritage experts. We, thus, do not investigate other information tasks, such as information exchange and information maintenance. Our main research questions are:

\footnotetext{
${ }^{1}$ We use the term Keeping up-to-date rather then the original term Browsing proposed by Kellar et al., to reflect the breadth of this task.

${ }^{2}$ Collectiewijzer is an online portal that supports information linking and exchange between cultural heritage institutions
}

1. Why do cultural heritage experts search? What is the motivation that gives context to their search activity?

2. Where do these experts search? Which sources do they use, why do they use them and do they experience any problems with them?

3. What are the experts' search tasks? What are typical search tasks, which search tasks do they do the most/least and do their tools sufficiently support the tasks?

To answer these questions we conducted a user study, which we describe in the following section.

\subsection{Procedure}

Most of the interviews took place at the participants' working environment. We conducted two pilot studies prior to the actual interviews to make sure all questions would be clearly understood. Each participant was interviewed individually with semi-structured questions and was asked to answer a questionnaire. The interview had three parts:

1. Introduction, demographic questions and informed consent.

2. Questions about the participant's main responsibilities and daily activities at work.

3. For each activity mentioned in part 2 , participants were asked to give examples and to describe the purpose of the activity, its frequency and the tools involved. Supplementary questions include their subjective impression based on their experience in using the tools.

After the interview, we asked them to demonstrate the tools they used and to give some examples on how they use them. On average, the whole interview took two to three hours. The interview was voice recorded; pictures and screen shots of the tools were taken. We analyzed the voice recording, photos and screen shots of the experts' tools and questionnaires. Activity descriptions (use cases) for every participant were noted down. Samples of screen shots of the tools from the participants helped us clarify the way our participants carry out their daily work and the problems that they face.

\subsection{Limitations}

We acknowledge that the method used has shortcomings. We did not capture the dynamics of experts' behavior over a longer time frame and relied on participants' (selective) memory. We may thus not have captured unconscious behavior of these experts. This study will also not reveal non work related search such as recreational search. However, we faced several restrictions: many experts are reluctant to give consent on automatic computer monitoring. This is not just because of privacy reasons, but also because it is against institute policy to share sensitive information, such as correspondence between experts, or install unauthorized monitoring software in the organization's computer network. These restrictions are the main reasons behind our pragmatic approach. Despite the approach that we took, we believe the study captured key aspects of the cultural heritage experts' information seeking behavior.

\subsection{Participants}

Based on recommendations from $\mathrm{ICN}^{3}$, we recruited experts who frequently search for information related to cultural heritage (see

\footnotetext{
${ }^{3}$ The Netherlands Institute for Cultural Heritage http://www.icn.nl/
} 
Table 1: Participants' Demography (total:17 people)

\begin{tabular}{|c|c|c|}
\hline & $\begin{array}{l}\text { Experience with digital libraries: } \\
\left.\text { Expert role: }{ }^{* *}\right) \\
\text { Cultural heritage sub-domain: } \\
\text { Age: years old } \\
\text { Affiliation: }{ }^{* *}\end{array}$ & $\begin{array}{l}\text { expert: } 3 \text {, intermediate: } 12 \text {, basic: } 2^{*)} \\
\text { researcher: } 6 \text {, curator: } 8 \text {, registrar: } 3 \text {, IT: } 3 \text {, teacher: } 2 \text {, student: } 3 \\
\text { ethnography: } 9 \text {, Dutch classic art: } 6 \text {, contemporary art: } 2 \\
\text { 21-30: } 3,31-40: 6,41-50: 3,51-60: 5 \\
\text { museum: } 8 \text {, company/freelance: } 2 \text {, university: } 5 \text {, cultural heritage institution: } 8\end{array}$ \\
\hline *) & $\begin{array}{l}\text { expert: } \\
\text { intermediate: } \\
\text { basic: }\end{array}$ & $\begin{array}{l}\text { IT experts, also manage the museum information systems. } \\
\text { Intensive use of search engines and online and offline digital libraries. } \\
\text { Minimal Internet/computer usage, e.g only email and Word. Prefer to use traditional libraries. }\end{array}$ \\
\hline **) & A single expert can have more then on & role and/or affiliation. \\
\hline
\end{tabular}

Table 1). In total, 17 Dutch professionals participated from 9 different cultural heritage institutions in the Netherlands (five museums, two companies, one university and ICN). Most participants use computers intensively at work. We interviewed experts with a variety of backgrounds to capture the different perspectives of information searching needs. Depending on the size of the organization, a single expert can have one or more roles. In large museums, an expert typically has a clearer and more specific role compared with smaller museums where an expert takes responsibility for several roles. We distinguish five expert roles in this study:

Researchers develop guidelines, recommendations, articles and books. Examples of cultural heritage research are developing different conservation techniques or developing theories on the history of acquisition of objects.

Curators are responsible for the collections and their documentation, including arranging loans, acquiring objects and planning exhibitions.

Registrars handle the digitization process of collections in the collection management system. Depending on the size of the museum, the registrars may work together with the curators in annotating collections. Together with curators, they also handle new entries and check whether the information is correct. They also prepare periodical reports on the museum collection status.

Teachers and students were recruited from a relevant Master's program at a Faculty of Art. The students are in their final year and carrying out their internship in a museum. The lecturers' main search activity is to prepare their course materials and keep up to date with the state of the art in the field. Students need to search regularly for making reports and assignments for their class. They also assist the researchers, curators and registrars while doing their internship.

IT personnel manage the museum database system. They prepare customized user manuals for the system and they often train and support other employees. In addition to this, they update the museum website, create regular reports on the status of collection documentation and assist registrars on collection documentation.

\subsection{Classification of use cases}

For every participant, we noted their main activities (i.e. use cases), which were further broken down into one or more sub-tasks. We filtered out the use cases that do not involve information seeking activities in the cultural heritage domain, such as project management or fund raising. In total, there are 17 participants with 53 use cases and 110 tasks. We then identified all information seeking tasks occurring within each use case. All information tasks were classified independently into one of the five information task categories by two reviewers. They were guided by information task descriptions similar to those given in the Related Work section. Cohen's kappa was used to measure the agreement between the two reviewers. We found the agreement of $\kappa=0.74$ (sufficient). The main disagreement between the two reviewers was in deciding

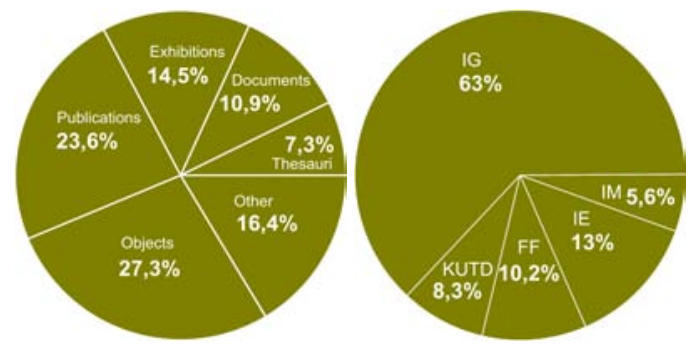

Figure 2: Types of experts' use cases (left). Types of experts' information seeking tasks (right).

which task category was more dominant for a given sub-task. This occurs for complex tasks, such as Information Gathering.

\section{USER STUDY RESULTS}

This section is divided into three parts, reflecting the three research questions of the user study. First, we discuss the experts' motivation that gives context to why they need to search for cultural heritage information. Second, we discuss the sources that the experts use to find information. Finally, we discuss the different types of experts' search tasks with examples.

\subsection{Why do experts search?}

We classified all use cases into five groups (see also the left of Figure 2):

1. Object handling. Experts need to gather information for the restoration, acquisition, loan or sale of an individual object. For example, before acquisition of a painting, a curator needs to research the history of the painting to see whether it is suitable for the museum's collection.

2. Exhibition planning. Experts spend a great deal of effort in research when preparing for an exhibition, e.g. finding interesting themes, carrying out comparison studies with previous exhibitions and publications. Serendipity is highly valued here. The main goal is to find different and interesting perspectives to present to the public.

3. Research for publication. Experts' publication activities can be divided into preparing publications for peer experts or for the general public. The first is mainly about developing guidelines, best practices, recommendations related to the cultural heritage domain and presenting new findings and discoveries. This activity also includes dissemination of the research through lectures and presentations. Publications for the general public are typically PR-related activities, such as writing an "object of the month" section for the website for which the history of the object, including interesting facts that could attract the general public, need to be found.

4. Managing the collections' documentation. Records in the col- 
Table 2: Frequency mention of source (total:204 mentions)

\begin{tabular}{rrr}
\multicolumn{3}{c}{ Source } \\
\hline \hline 1 & Literature & $21.4 \%$ \\
2 & Archives and catalogs & $18.9 \%$ \\
3 & Personal contact & $10.2 \%$ \\
4 & Visit exhibition/museum & $1.5 \%$ \\
\hline & Total offline sources: & $\mathbf{5 2 . 0 \%}$ \\
\hline \hline 5 & Reputable website & $18.9 \%$ \\
6 & Collection management system & $13.8 \%$ \\
7 & Search engine & $8.7 \%$ \\
8 & Other digital sources & $6.6 \%$ \\
\hline & Total digital sources: & $\mathbf{4 8 . 0 \%}$ \\
\hline
\end{tabular}

lection management system are constantly updated by curators and registrars. For example, new objects need to be registered, information about old collections needs to be updated. When a new object is registered, the expert needs to compare annotations of similar objects and search for more descriptions by looking further in various sources.

5. Building thesauri. Thesauri are controlled vocabularies used, in this case, for annotating objects in museum records. Within an organization, it is important that everyone use the same terms to express the same concepts in the museum records. So experts need to collect terms important for the field from selected sources such as literature, dictionaries, library archives and object descriptions for inclusion in their thesauri. Information from reputable websites, such as those of other museums or cultural heritage organizations, are also used. Typically, multiple experts need to agree on the proposed terms before they are included in the thesaurus. Different cultural heritage branches may use different thesauri. Examples mentioned by our experts are the $\mathrm{SVCN}^{4}$ thesaurus for Dutch ethnography and the AAT/AATNED ${ }^{5}$ for general art and architecture terms.

\subsection{Where do experts search?}

Experts consult a large variety of sources to look for the answers they need (see Table 2 and 3 for details). Source credibility is an important aspect for experts. For research, they carefully select and refer only to reliable sources.

1. Literature: This includes magazines, dictionaries, books, publications, biographies, encyclopedias, journals and reference databases such as RKD library ${ }^{6}$ or Picarta ${ }^{7}$. Offline literature remains the most important source for experts because comprehensive knowledge on art, culture and history are usually in books and not yet available in digital form. Online integrated bibliographical search, such as Picarta, helps experts in their search for the correct literature across many libraries.

2. Archives and catalogs: This includes exhibition catalogs, auction logs, inventory cards and remarks fields made by curators. Old documention about objects, often not yet digitalized in the collection management system, are stored as inventory cards and remarks on log books.

3. Personal contact: Personal contact and networking remain one of the most important means of seeking information. For example, communication between curators is about each other's collection or to find out more about a particular historical or cultural topic.

\footnotetext{
$4_{\text {http: / / www.sven.nl/ }}$

5 http://www.getty.edu/research/conducting \_research/

4. Visit exhibitions or museums: Experts gain knowledge by conducting working visits to other museums to see their collections in person.

5. Reputable Internet sources: It is extremely important for experts to use reliable information sources, including sources on the Internet. The museum curators, who are responsible for the annotation of their objects, agree on which online resources meet their quality standards. Reputable Internet sources are, for example, specific museums websites, cultural heritage institution websites (SVCN website ${ }^{8}$, RKD online, galleries.nl), global gazetteer ${ }^{9}$ and the CIA fact book ${ }^{10}$.

6. Collection management system Each museum maintains its own information system. This stores the records of all objects and is often an integrated system to help museum employees with almost all aspects of their work, such as management, status report generation and loan request processing. Examples of commonly used commercial systems are $\mathrm{TMS}^{11}$ and $\mathrm{ADLIB}^{12}$.

7. Search Engines: Most experts mentioned Google as their search engine. In contrast with how experts use reputable websites, experts use search engines for navigational search (e.g., to find an artist, museum or gallery website) and, interestingly, to seek inspiration (e.g. "what does Google have on Iranian Calligraphy?").

8. Other digital sources: This includes all tools not mentioned above, such as online newspapers or RSS feeds.

Table 2 shows a summary of the sources used by the participants. Figure 3 shows the interfaces of these sources. We can see that the number of use cases using traditional sources (1-4 add up to $52 \%$ ) and digital sources (5-8 add up to $48 \%$ ) is comparable. While more and more sources are made available for online search, offline literature remains a very important source of cultural heritage information.

\subsection{What are the experts' search tasks?}

We originally hypothesized that information seeking tasks are dependent on the expert role. The analysis, however, suggested otherwise: tasks depend more on the type of use case. This is because most of the use cases mentioned by the experts are done in team work by multiple expert roles. Consequently, some of the use cases are overlapping. For example, management of collection documentation is done by curators and registrars, and student interns help curators with their daily work.

We have thus classified the use cases into different task categories of Figure 1. The breakdown of each task is summarized in Table 4 and the right of Figure 2). We discuss each information seeking task: fact finding, information gathering and keeping up-to-date, and illustrate each task with examples given by our participants.

\subsubsection{Fact Finding}

Fact Finding search questions vary from simple to very complex. Typical examples of simple queries include:

"What is the contact information of the gallery?" [P4]

"To which tribe/culture does this object belong?" [P1]

"From where does this object originate?" [P3]

Complex queries typically combine several constraints. For example, a curator was given an assignment to select and lend several paintings from the collection for a government building. She

\footnotetext{
8 http: //www.sven.nl/

9 http://www.allm-geodata.com/productsl.htm

10 https://www.cia.gov/library/publications/

the-world-factbook/

11 http://www.gallerysystems.com/products/tms.html

12 http: //www.adlibsoft.com/
} 
Table 3: Distribution of sources for different types of use cases (total:204 items)

\begin{tabular}{|c|c|c|c|c|c|c|c|c|c|c|c|c|c|c|c|c|}
\hline Use case type vs. Source & \multicolumn{2}{|c|}{ Books } & \multicolumn{2}{|c|}{ Rep.Website } & \multicolumn{2}{|c|}{ Archive } & \multicolumn{2}{|c|}{ Search Eng } & \multicolumn{2}{|c|}{ Museum DB } & \multicolumn{2}{|c|}{ Person } & \multicolumn{2}{|c|}{ Other } & \multicolumn{2}{|c|}{ Visit Exh.Museums } \\
\hline 1.Objects & 9 & $19.6 \%$ & 9 & $23.7 \%$ & 11 & $28.2 \%$ & 3 & $17.6 \%$ & 8 & $28.6 \%$ & 4 & $20 \%$ & 1 & $7.7 \%$ & 1 & $33.3 \%$ \\
\hline 2.Exhibitions & 9 & $19.6 \%$ & 7 & $18.4 \%$ & 10 & $25.6 \%$ & 4 & $23.5 \%$ & 3 & $10.7 \%$ & 3 & $15 \%$ & 1 & $7.7 \%$ & 1 & $33.3 \%$ \\
\hline 3.Publications & 17 & $37 \%$ & 10 & $26.3 \%$ & 8 & $20.5 \%$ & 4 & $23.5 \%$ & 7 & $25 \%$ & 6 & $30 \%$ & 8 & $61.5 \%$ & 1 & $33.3 \%$ \\
\hline 4.Documentation & 4 & $8.7 \%$ & 2 & $5.3 \%$ & 2 & $5.1 \%$ & 1 & $5.9 \%$ & 7 & $25 \%$ & 0 & $0 \%$ & 0 & $0 \%$ & 0 & $0 \%$ \\
\hline 5.Thesauri & 4 & $8.7 \%$ & 7 & $18.4 \%$ & 1 & $2.6 \%$ & 2 & $11.8 \%$ & 2 & $7.1 \%$ & 1 & $5 \%$ & 1 & $7.7 \%$ & 0 & $0 \%$ \\
\hline 6.Other & 3 & $6.5 \%$ & 3 & $7.9 \%$ & 7 & $17.9 \%$ & 3 & $17.6 \%$ & 1 & $3.6 \%$ & 6 & $30 \%$ & 2 & $15.4 \%$ & 0 & $0 \%$ \\
\hline Total & 46 & & 38 & & 39 & & 17 & & 28 & & 20 & & 13 & & 3 & \\
\hline
\end{tabular}

Table 4: Distribution of the experts' information seeking tasks (total:110 use cases)

\begin{tabular}{|c|c|c|c|c|c|c|c|c|c|c|c|c|c|c|}
\hline \multicolumn{2}{|l|}{ Search task vs. Use case type } & \multirow{2}{*}{$\begin{array}{c}\text { Total } \\
11\end{array}$} & \multicolumn{2}{|c|}{ Thesauri } & \multicolumn{2}{|c|}{ Documents } & \multicolumn{2}{|c|}{ Exhibitions } & \multicolumn{2}{|c|}{ Publications } & \multicolumn{2}{|c|}{ Objects } & \multicolumn{2}{|c|}{ Other } \\
\hline Fact finding (FF) & $10.2 \%$ & & $\overline{0}$ & $0 \%$ & $\overline{11}$ & $8 \%$ & 0 & $0 \%$ & $\overline{5}$ & $16 \%$ & 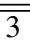 & $12 \%$ & 2 & $13 \%$ \\
\hline Information Gathering (IG) & $63.0 \%$ & & & & & & & & & & & & & \\
\hline 1.Comparison & & 9 & 0 & $0 \%$ & 2 & $17 \%$ & 0 & $0 \%$ & 2 & $7 \%$ & 3 & $12 \%$ & 2 & $13 \%$ \\
\hline 2.Relationship search & & 6 & 0 & $0 \%$ & 0 & $0 \%$ & 2 & $10 \%$ & 1 & $3 \%$ & 1 & $4 \%$ & 2 & $13 \%$ \\
\hline 3.Topic search & & 39 & 4 & $57 \%$ & 4 & $33 \%$ & 7 & $35 \%$ & 10 & $32 \%$ & 8 & $32 \%$ & 6 & $40 \%$ \\
\hline 4.Combination & & 9 & 0 & $0 \%$ & 1 & $8 \%$ & 3 & $15 \%$ & 3 & $10 \%$ & 2 & $8 \%$ & 0 & $0 \%$ \\
\hline 5.Exploration & & 7 & 0 & $0 \%$ & 0 & $0 \%$ & 5 & $25 \%$ & 1 & $3 \%$ & 1 & $4 \%$ & 0 & $0 \%$ \\
\hline Keeping Up-to-date (KUTD) & $8.3 \%$ & & & & & & & & & & & & & \\
\hline 1.Active & & 7 & 1 & $14 \%$ & 1 & $8 \%$ & 0 & $0 \%$ & 1 & $3 \%$ & 4 & $16 \%$ & 0 & $0 \%$ \\
\hline 2.Passive & & 2 & 0 & $0 \%$ & 0 & $0 \%$ & 0 & $0 \%$ & 1 & $3 \%$ & 0 & $0 \%$ & 1 & $7 \%$ \\
\hline Information Maintenance (IM) & $5.6 \%$ & 6 & 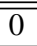 & $0 \%$ & 2 & $17 \%$ & 1 & $5 \%$ & 2 & $7 \%$ & 1 & $4 \%$ & $\overline{0}$ & $0 \%$ \\
\hline Information Exchange (IE) & $13.0 \%$ & & & & & & & & & & & & & \\
\hline 1.Transaction & & 2 & 0 & $0 \%$ & 1 & $8 \%$ & 1 & $5 \%$ & 0 & $0 \%$ & 0 & $0 \%$ & 0 & $0 \%$ \\
\hline 2.Communication & & 12 & 2 & $28 \%$ & 0 & $0 \%$ & 1 & $5 \%$ & 5 & $16 \%$ & 2 & $8 \%$ & 2 & $13 \%$ \\
\hline
\end{tabular}

needed to select several paintings with appropriate themes and sizes for the building, that would fit on a wall having certain space constraints: "Are there paintings from our collection, either depicting Amsterdam or created by a painter from Amsterdam, with a width smaller than $50 \mathrm{~cm}$ ?" [P6]

\subsubsection{Information Gathering}

With 63\%, Information Gathering tasks dominate our expert's use cases. Based on the similarities between the use cases within this group, we identified the following sub-tasks: (See also Table 4):

1. Comparison involves gathering information to compare differences and similarities between objects or sets of objects. For example: a curator needs to make an acquisition proposal each year. To do this, she needs to make an assessment of the objects currently in their collection and in that of others: "What objects from the Middle-East do other museums in the Netherlands have? Is there any tribe or region not represented in our collection or in the collection of other museums? If there is, we need to find out exactly what kind of object we should get." [P14]

2. Relationship search is about finding relationships between individual pieces of information. For example, a curator needed to research the network of people around the Dutch painter Rembrandt van Rijn. To do this, she performs a literature study, searches for close and distant family members of the artist, the mostly rich and influential people whom he had portrayed, the people whom he had met socially and been friends with. The questions asked about these people are the same every time: "Who is this person, what does s/he do and what is the nature of his/her relationship with Rembrandt?" [P8]

3. Topic search queries can typically be formulated as "Tell me more about ..." questions. For example: one of our curator's responsibilities is to maintain the descriptive labels that are associ- ated with all objects in the collection. Among the objects is a specific Jewish ceremonial coat. To determine the history of this coat, the curator checks literature, newspaper archives, auction records, etc.: "Where and when was this coat made? Was there any restoration done to the coat? What is the purpose of the coat? What does it symbolize? Is there any meaning behind the embroidery? Where was it used? Who used it? Was it ever used in an important historical event?" [P8]

4. Exploration or exploratory search, is typically not goal directed. Instead, the expert may associatively follow one train of thought after another. For example, one of our experts was looking for art suitable for decorating the staircase area of a public space. Given that "staircase art" is not an established genre, the expert knows that searching on this term directly will not provide the intended results. Instead, the expert looks for related projects for suggestions, such as artists who do landscaping or city planning art projects: “... On specific situations, (such as) in the Staircase project, I look a lot at similar examples of artworks in staircases, for instance, art projects connected to landscaping or city planning, something like that. " [P4]

5. Combination is about finding matches among pieces of information, most likely from different sources. This task is similar to fitting pieces of a puzzle together to see the bigger picture. For example, a new part of a public building needs to be decorated and the client (the government) has assigned an art adviser to make the planning. The art adviser first gathers the requirements for a public-art piece such as the amount of space, the preferences of the client, the purpose of the building, the theme of the art and the environment. The art adviser then searches on public-art artists and compares their portfolios containing examples of their work. The next step is to match the collected requirements with the artists. The art adviser needs to make a selection of several artists and then 

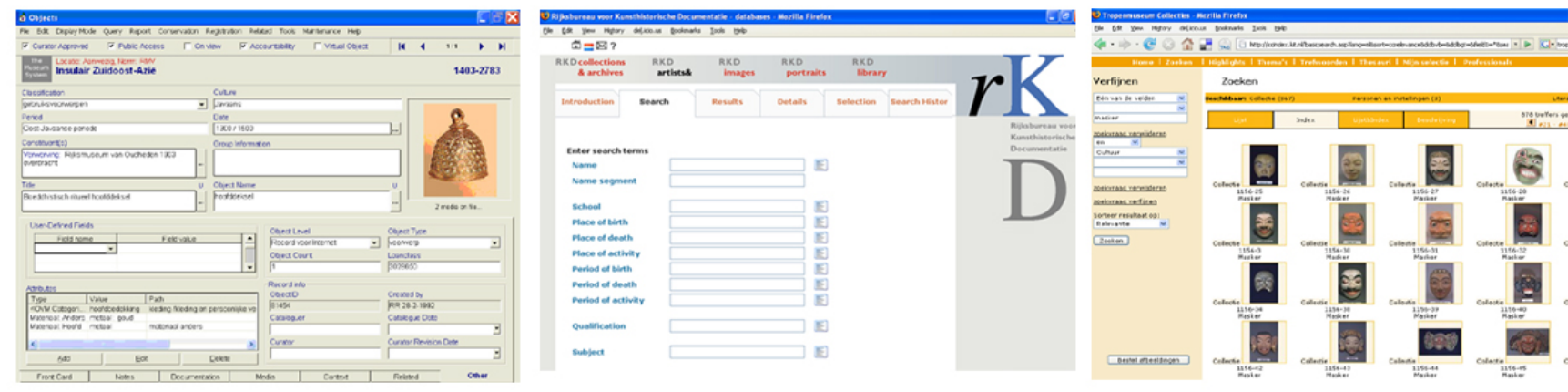

Figure 3: Interface screen shots from different types of sources, giving an impression of the typical complexity of the search interface: TMS collection management system (left), RKD online library (middle), Tropenmuseum's public website (right).

present these options to the client for approval: "Which public-art artists match the project requirements?" [P4]

\subsubsection{Keeping Up-to-date}

There are two ways in which the experts keep up-to-date: by actively seeking for updates, or passively, using technology that automatically detects content updates and sends notifications.

1. Active: Going to the sources and scan through for changes from sources (e.g. browsing). Experts keep up with the latest news on artworks of their interest, follow auction news, keep up-to-date with the price of artworks or with changing artwork ownership.

2. Passive: Using technology to automatically deliver new information from sources (e.g. RSS feed, email). Many experts subscribe to community mailing-lists to receive information on developments such as new exhibition announcements or reviews of new books and other publications.

\section{DISCUSSION}

The results in the previous section allowed us to identify the experts' most important information seeking tasks. In this section, we identify a number of problems with using current tools, categorized by information seeking task. Furthermore, from our observations, we distill general trends of the information seeking needs in the cultural heritage domain that may extend to other domains.

\subsection{Fact Finding}

Experts' search questions can be simple or complex, with many constraints. Most search applications they use support both simple and complex search. The two most frequent Fact Finding problems are where simple search does keyword matching across all descriptions and returns too many results; and where advanced search specifies values as constraints and retrieves too few or no results. Difficulties in building queries can occur if the expert is not familiar with the correct controlled vocabularies from the thesauri that are used to describe the objects.

\subsection{Information Gathering}

More than half $(63 \%)$ of the use cases we found can be classified as Information Gathering tasks. Many of these use cases are relatively complex when compared to Fact Finding use cases, and they are often related to activities such as preparing new publications, designing exhibitions or managing collection documentation (see table 4). Information Gathering contains very distinct use cases, which can be further classified as sub-tasks such as comparison, relationship, topic, combination and exploration search tasks. Topic search is the main type of search for experts that need to prepare exhibitions, write publications or document objects. In Information Gathering tasks, experts typically search with several different sources. They are forced to manually collect, examine and synthesize relevant pieces of information, because these higher level activities are not supported by their tools. For each Information Gathering sub-task, we give examples that illustrate this mismatch and describe how experts compensate for the lack of tool support: 1. Comparison. Current tools are often not geared towards comparing two objects side by side, and comparing sets of objects is an even harder task: "For our exhibition, what objects from Aceh" that are missing in our own collection can we borrow from that museum?" [P3]

When a curator would like to compare parts of her collection with that of another museum, she might prefer to pick up the phone and discuss the issue with the curator of the other museum directly. Curators report this is often more effective than trying to browse the other museum's collection website, especially when both curators know their collection by heart. However, relying solely on a curator's memory may not be wise for large collections: As one curator explains after the interview: "For my own collection, there are around ten thousand objects, it is still possible to remember my own stuff, but I cannot imagine a curator remembering every detail if he has to take care of hundreds of thousands of objects." [P15] In such cases, experts are forced to look up the relevant objects themselves, and to do all the higher level comparison related tasks manually as these are not supported by their tools.

2. Relationships Search. In the example of the Rembrandt exhibition, our expert executed a comprehensive search for relationships between Rembrandt and other people, to create a story for the exhibition. In such cases, an expert typically consults many sources, such as art books, history books, birth records and biographies. She takes notes of different names from one book, and some of these names may refer to the same person that are mentioned under another name in another source. She then has to cross reference to see if there is more information about the related persons found in other sources. "I check old archives, history books, collect the names and make the connections." [P8]

Finding such relationships requires a lot of time and energy. Sometimes the relationship is direct and easily found within one source. More often, however, the relationship is indirect, requiring searching through many sources and making interpretations along the way. These tasks have to be done manually because they are not supported by the expert's tools.

3. Topic Search. When an expert needs to find out everything

\footnotetext{
${ }^{13}$ Aceh is a region in Sumatra, Indonesia.
} 
s/he can about an individual object, e.g. when it is to be added to the collection, many online and offline sources need to be consulted. There is no direct way to obtain all the answers. Most search interfaces provide only keyword search, and rarely allow users to browse by topic or obtain suggestions for related results. One participant wanted to know, "Are there any objects in the museum related to the African trade in the 17th century theme? [P17]". She tried many related keywords in combinations that she thinks may be included in the description of the object: 'trading africa', 'goudkust' (name of area), 'handel' (trade), '1799', 'west-africa', 'akan' (name of people who lived in the area), 'elmina' (name of a fort), 'weight', 'boeien' (chain). Eventually she found a few carved metal weights that the traders used with a balance. She was quite sure that there should be many more objects about that topic but she could not find them. "There should be chains and special boxes with ornaments (to put gifts in), but I couldn't find them." [P17]

For topic search, simple and advanced search interfaces (see figure 3) are not sufficient. Users have to (almost randomly) guess related keywords, which is unlikely to lead to finding all desired answers.

4. Exploration. When experts need to do exploratory search, they rely heavily on their domain expertise. Similar to topic search, users will try out different terms, but only because this is what the search interface supports. The difference is that they try to be more exploratory with the chosen terms in the hope that they will obtain results serendipitously. Currently, experts are forced to rely on their cognitive skills and creativity to bring all this knowledge together. In the "Staircase" example, the expert tried different terms that she thinks will lead to promising results (e.g. staircase art, landscape art, city planning art). These terms are usually very specific, relying heavily on the expert's creativity, and the procedure itself is often based on trial and error and may lead to no result. Exploratory search is helpful when the expert is looking for new ideas, e.g. for an exhibition. The problem is that, while some tools may support browsing from one link to another, none supports the exploratory search task directly.

5. Combination. Finding a match between two pieces of information is challenging. For example, an expert needs to find an artist, who best matches the project requirements, from a list of candidates. She selected several artists who were potential candidates, collected samples of their work and presented her findings to the client. "I select around 5 best artists who I think are suitable for the job, then I collect and present their portfolio to the client" [P4]. Much of the work involved in the combination task is done manually and relies heavily on the expert's experience and personal judgment. The problem is that this task requires diligence and takes large amounts of time and effort.

\subsection{Keeping Up-to-date}

Even though our participants did not mention this activity as a priority, they would like to be kept informed of cultural heritage news that is interesting for them, such as new exhibitions, new publications, social and professional events. Passive KUTD (e.g. being subscribed to a mailing-list or RSS feed) takes less effort than active KUTD (e.g. browsing websites) because experts receive notification only when there are changes. Our study suggests, however, that the usage of passive KUTD is low. The reason behind this is because not all systems provide support for passive KUTD and experts are not used to passive KUTD - this was mentioned only twice out of 110 use cases. Despite its utility, passive KUTD can be irritating, especially when changes are too frequent or insufficiently important, resulting in information overload. "Well, I am subscribed to a mailing-list but I do not have time to read all those emails (laugh)." [P5]. As a result, only few experts feel the need to subscribe to mailing lists or RSS feeds. The problem is in finding a balance between providing the experts with the most recent and relevant information while not letting them feel overwhelmed by too much information.

\subsection{General Trends}

Even though we focus our study on the cultural heritage domain, we found several issues that indicate general trends in information seeking behavior that may be relevant and apply to other domains, such as humanity, social sciences and professionals [2, 14, 17, 20].

\section{Information Seeking Tools}

We observe that the current tools used by experts do not fully support fact finding, information gathering and keeping up-to-date information seeking needs. We identify several reasons why this is: (a) difficulty in making complex queries (b) pieces of information need to be collected from many different sources before being synthesized by experts (c) data is mostly unstructured, making the retrieval process challenging.

Fact-finding requests require better data descriptions and tools that assist users in expressing complex queries. For most of the information gathering tasks, we feel current tools are insufficient because the tasks often rely on finding higher level relationships between individual facts, which are distributed across heterogeneous sources. Typical examples are tasks that require a combined view on the collections of two separate museums or tasks that combine information from the museum's collection management system with more general art-historic background information. In these cases, tools that can make the higher level relationships explicit are needed. Similar findings were reported by [20], for humanity researchers where the search tools available are not adequate for their complex information seeking needs.

\section{Searching Multiple Sources}

In most use cases, experts need to consult multiple sources to obtain their answers (see Table 3). This is consistent with research found in other domains such as in $[14,17]$. It is rarely the case that experts rely on only one source of information. For example, in Information Gathering tasks, experts need to constantly compare, relate, combine and explore information from different sources. Even though none of the experts complained about the tedious way of searching, we observe that they spend large amounts of time and effort on repetitive searching because they need to repeat the same query in different sources.

\section{Communication}

While experts consult multiple online and offline sources, personal contact between experts remains an important means of obtaining information (12 out of 14 information exchange use cases). We identify a number of reasons for this. Experts find it more convenient to contact other experts rather than to do the searching themselves; other experts have knowledge and experience that cannot be found in any document or information source; experts need to consult each other to find consensus on certain matters or decisions.

"We have regular meetings with other museums to decide which words should be in the thesaurus.” [P2]

When asked about how she planned her exhibition: “...I talk to many people who are experts in Rembrandt." [P6].

\section{Provenance and Trust}

Another important issue mentioned by our experts in this context is source credibility: experts only mine information from sources 
they trust. This is also the case in other domains [14]. Generally these trusted sources are agreed upon within a specific community.

"For the thesaurus we decided to use all the literature which we agreed upon. Several years ago we did not accept anything from Internet, but nowadays some. The curators are a little bit afraid of it, who says the information is correct?"[P2]. When systems use information from multiple sources, source credibility measure needs to be taken into account at both a functional and interface level to help users decide on the quality of the search results.

\section{Information Maintenance}

While our study focuses on understanding search behavior, we found that information seeking behavior is closely related to information maintenance. Once an expert finds a piece of information, she may want to store it because it may be reusable in the future. While cultural heritage experts adopt many strategies commonly used also by other professionals [2], such as making bookmarks and keeping notes, there is no integrated way to store and maintain retrieved information from different sources that enables experts to re-access information efficiently. Thus, if we want to support these experts' information seeking tasks, we also need to help these experts maintain the information once discovered.

\section{DESIGN IMPLICATIONS}

The results of our study suggest that cultural heritage experts need better support for complex query formulation, information gathering tasks, keeping up-to-date and for searching across multiple sources. In this section, we discuss potential research directions and the impact of this study on the design of the search tools developed within the MultimediaN project.

\section{Information Seeking Tools}

Fact Finding - Our results show that experts would benefit from explicit support for formulating complex queries. A facet browser [6, 21] is an example of an interface that can assist users in building complex queries in an incremental manner. The interface shows intermediate results as the user builds a complex query step by step. Yee et al. showed that facet interfaces work well for visual resources described by structured data [21]. Cultural heritage information is often visual (e.g. photographs of museum objects, visual archives and photos of artists) and stored in structured museum databases. Deployment of a facet browser interface, however, typically requires extensive configuration of data and software. This requirement makes facet browsing less compelling for museums, which often use more generic museum management systems. Facet browsers also may not work well on heterogeneous datasets because the number of facets tends to become too large. /face $\mathrm{f}^{14}$ is a facet browser that we developed for heterogeneous domains [6]. We are currently researching how we can effectively use facet browsing within cultural heritage organizations.

Information Gathering - Due to the complexity and diversity of information gathering search activities, different specialized search tools may be required. For example, to support experts searching for relations among artefacts or concepts, the sources used need to be structured and linked to each other, providing relationships among museum entities, such as between artists, art styles and artworks. Our Relation Search ${ }^{15}$ prototype allows users to find connections between any two objects or concepts, such as between an artist and an artwork or between two artists. The results are orga-

\footnotetext{
14 http://e-culture.multimedian.nl/demo/facet
}

15 http://e-culture.multimedian.nl/demo/path nized from the shortest (showing direct relations) to the longest (showing indirect relations). Current challenges in this type of search is on how to differentiate the "interesting" from the large amount of "trivial" relations, since these notions are subjective and context dependent.

Another example is topic search. Whenever experts search for information centered around a particular topic, they need information related to a single term as well as suggestions for related terms, e.g. nearby geographical locations or related cultures. While recommender systems based on content-based and collaborative filtering have already been developed for suggesting relevant research papers $[10,18]$, further research is needed to explore if and how such approaches can deployed in the domain of cultural heritage.

Keeping Up-to-date - While experts need to keep up-to-date with the development of particular topics, they should not be overwhelmed with irrelevant information. User interfaces developed for this purpose, such as RSS readers, already exist. Our study, however, shows that only few of our experts have actually used them. This may be because RSS readers are relatively new, and over time more domain experts may start to use them. One thing that may be helpful for subscribers is a tool to manage the frequency of update and provide filtering and/or ranking based on, e.g., priority and topic. Further research is needed to explore to what extent keeping upto-date functionality can and should be integrated into tools that experts already use.

\section{Searching Multiple Sources}

For many experts, a "meta-search" functionality that simply aggregates search results from multiple sources would already be a great improvement over the current practice of repeating the same query in isolated search applications. Our project's demonstrator [15] allows users to search and navigate multiple collections from multiple organizations. In addition, our system uses available domain background knowledge to find semantic relationships between items from different collections. We are currently researching how to effectively explore these relationships in both the search algorithm and in the presentation of the results.

\section{Provenance and Trust}

Trust in the information source is a key aspect for experts, so applications providing access to multiple sources need to explicitly communicate the origin of all information to the user. How provenance can best be conveyed when information from many sources needs to be displayed is still an open research question. As a first step, we conducted a study on how visualization of the credibility of the cultural heritage sources influences peoples' decisions [22]. This study showed, for example, that visualization of source credibility ratings significantly increased the confidence of users that were presented with information from multiple sources. Additionally, trust measures could also play a role in the search functionality itself, for example by ranking results based on the credibility of their source. Further research is needed on the design and evaluation of this type of functionality.

\section{CONCLUSIONS AND FUTURE WORK}

We have presented the results of our study on the information seeking needs of cultural heritage experts. The goal of the study is to understand: why do cultural heritage experts search; where do they search for information; and what are their information seeking tasks. Our study suggests that experts' daily search tasks are dominated by (high level) Information Gathering while the search systems they use support (low level) Fact Finding tasks. As a result, 
experts need to compare, relate and combine pieces of information manually or ask their colleagues. We also found that while the experts have simple as well as complex questions, their current tools provide insufficient interface support for query formulation. In addition, most experts' search tasks require information from many different sources, while their tools tend to support search in only one source at a time. Finally, we discussed the study's implications on the design of the search tools developed in our project.

Our next step is to concentrate on each of the tasks and see how to improve the search experience. We will focus our attention mostly on Information Gathering, since this is our experts' main search activity but seems to have the least support. We will investigate the different ways to present cultural heritage information that enable our experts to compare, relate, explore and combine information and search for related topics. Furthermore, we plan to perform user evaluations on our proposed interfaces to verify that we have indeed helped our experts in their search.

\section{ACKNOWLEDGMENTS}

We thank the participants from Amsterdam Tropenmuseum, Dutch National Museum of Ethnology Leiden, Rijksmuseum Amsterdam, Afrika Museum Berg en Dal, Jewish Historical Museum Amsterdam, DEN and ICN for for taking the time from their busy schedules to share their experiences with us. We thank M. F. van Oorsouw, A. Teesing, M. de Niet, L. Rutledge, L. Aroyo, M. Hildebrand, the MultimediaN E-Culture project members and other CWI colleagues for their help in this study. This research was supported by the MultimediaN project funded through the BSIK programme of the Dutch Government and by the European Commission under contract FP6-027026, Knowledge Space of semantic inference for automatic annotation and retrieval of multimedia content K-Space.

\section{REFERENCES}

[1] A. Broder. A taxonomy of web search. SIGIR Forum, 36(2):3-10, 2002.

[2] H. Bruce, W. P. Jones, and S. T. Dumais. Information behaviour that keeps found things found. Inf. Res., 10(1), 2004.

[3] D. O. Case. Looking for Information, Second Edition: A Survey of Research on Information Seeking, Needs, and Behavior (Library and Information Science). Academic Press, Dec 2006.

[4] C. W. Choo, B. Detlor, and D. Turnbull. A behavioral model of information seeking on the web: preliminary results of a study of how managers and it specialist use the web. In ASIS Annual Meeting Contributed Paper, 1998.

[5] C. W. Choo, B. Detlor, and D. Turnbull. Web Work Information Seeking and Knowledge Work on the World Wide Web. Kluwer Academic Publishers, 2001.

[6] M. Hildebrand, J. van Ossenbruggen, and L. Hardman. /facet: A Browser for Heterogeneous Semantic Web Repositories. In Proc of ISWC'06. vol. 4273/2006 of LNCS, p. 272-285. Springer Berlin / Heidelberg, 2006.

[7] M. Kellar, C. Watters, and K. M. Inkpen. An exploration of web-based monitoring: implications for design. In Proc of CHI'07, p. 377-386, New York, NY, USA, 2007. ACM Press.
[8] M. Kellar, C. Watters, and M. Shepherd. A field study characterizing web-based information seeking tasks. Technical report, Dalhousie University Halifax, 2005.

[9] M. Kellar, C. Watters, and M. Shepherd. The impact of task on the usage of web browser navigation mechanisms. In Proc of GI'06, p. 235-242, Toronto, Ont., Canada, 2006. Canadian Information Processing Society.

[10] S. M. McNee, I. Albert, D. Cosley, P. Gopalkrishnan, S. K. Lam, A. M. Rashid, J. A. Konstan, and J. Riedl. On the recommending of citations for research papers. In Proc of CSCW'02, p. 116-125, New York, NY, USA, 2002. ACM Press.

[11] J. B. Morrison, P. Pirolli, and S. K. Card. A taxonomic analysis of what world wide web activities significantly impact people's decisions and actions. In Proc of CHI'01, p. 163-164, New York, USA, 2001. ACM Press.

[12] P. L. T. Pirolli. Information Foraging Theory: Adaptive Interaction with Information. Oxford University Press, 2007.

[13] D. E. Rose and D. Levinson. Understanding user goals in web search. In Proc of WWW'04, p. 13-19, New York, NY, USA, 2004. ACM Press.

[14] D. K. Rosner, J. M. Josling, A. Moed, and E. Oreglia. Understanding target users of a digital reference library. In Proc of JCDL'07, p. 495-495, New York, NY, USA, 2007. ACM.

[15] G. Schreiber, et al. Multimedian e-culture demonstrator. In I. Cruz, S. Decker, D. Allemang, C. Preist, D. Schwabe, P. Mika, M. Uschold, and L. Aroyo, editors, In Proc of ISWC'06, vol. 4273 of LNCS, p. 951-958. Springer, 2006.

[16] A. J. Sellen, R. Murphy, and K. L. Shaw. How knowledge workers use the web. In Proc of CHI'O2, p. 227-234, New York, NY, USA, 2002. ACM Press.

[17] H. R. Tibbo. Primarily history: historians and the search for primary source materials. In Proc of JCDL'02, p. 1-10, New York, NY, USA, 2002. ACM. Press.

[18] R. Torres, S. M. McNee, M. Abel, J. A. Konstan, and J. Riedl. Enhancing digital libraries with techlens+. In Proc of JCDL'04, p. 228-236, New York, NY, USA, 2004. ACM Press.

[19] M. van der Graaf. Behoefteonderzoek gebruikers collectiewijzer. Available at: http://project.collectiewijzer.nl/ images/stories/pdf/

Behoefteonderzoek_gebruikers_Collectiewijzer.pdf, Dec. 2006.

[20] C. Warwick, J. Rimmer, A. Blandford, and G. Buchanan. User centred interactive search in the humanities. In Proc of JCDL'05, p. 400-400, New York, NY, USA, 2005. ACM. Press.

[21] K. P. Yee, K. Swearingen, K. Li, and M. Hearst. Faceted metadata for image search and browsing. In Proc of CHI'03, p. 401-408, Ft. Lauderdale, Florida, USA, 2003. ACM Press.

[22] J. Zhang. Finding cultural heritage information from multiple and diverse sources in semantic web applications: The effect of visualizing source credibility. Master's thesis, University of Amsterdam, 2007. 